\title{
HANDOFF NOTIFICATION IN WIRELESS HYBRID NETWORKS
}

\author{
Guillaume Chelius \\ Inria Ares Team, Laboratoire Citi, Insa de Lyon, \\ 21, avenue Jean Capelle, 69621 Villeurbanne Cedex, France \\ Guillaume.Chelius@insa-lyon.fr \\ Claude Chaudet \\ Inria Ares Team, Laboratoire Citi, Insa de Lyon, \\ 21, avenue Jean Capelle, 69621 Villeurbanne Cedex, France \\ Claude.Chaudet@insa-lyon.fr
}

\begin{abstract}
Hybrid networks composed of a wireless infrastructure network providing Internet access to an underlying ad hoc network are more and more attractive due to their low installation cost. In these all-wireless environments, performance is a key issue as radio bandwidth is scarce. Handoffs management is particularly important as these networks are likely to be highly mobile. Mobility notification should therefore be optimized in order to limit signaling overhead while keeping a good reactivity against terminals mobility. This article presents and studies by simulation different level optimizations applied to a modified Cellular IP protocol.
\end{abstract}

Keywords: ad hoc networks, hybrid networks, micro-mobility

\section{Introduction}

Wireless communications have to play a crucial role in computer networks. They offer open solutions to provide mobility and services where the installation of a complex wired infrastructure is not possible. With the exponential growth of wireless communications, a wide range of wireless devices has been released. In the same time, the number of cellular phones has significantly increased. The Internet becomes pervasive and is now bound to cellular networks. Research on wireless networks has roughly been concentrating on two distinct themes. The first one aims at extending the edge of infrastructure networks by the integration of a last wireless hop. The radio connectivity is provided by Base Stations at the edge of the network. The second theme 
concerns infrastructure-free and auto-organized wireless networks: Mobile Ad hoc Networks (MANet).

Research efforts aiming at merging cellular wireless and ad hoc networking have recently increased $[4,10,5,7-1,6]$. Hybrid networks, the extension of cellular networks using ad hoc connectivity, offer obvious benefits. On one hand, they the extend cellular network coverage using ad hoc connectivity and on the other hand they provide a global Internet connectivity to ad hoc nodes. However, deployment of a wired cellular infrastructure still induces a high cost as well as a lot of constraints. Both costs and constraints can be reduced if we replace the wired infrastructure network by a fully wireless one. The infrastructure network becomes a collection of static wireless nodes acting both as Base Stations and infrastructure routers. Infrastructure communications become wireless and multi-hop. As the wireless medium slightly differs from the wired one, the design of classical micro-mobility protocols must be rethought and if necessary modified.

In this article, we study how ad hoc node mobility/handoffs must be notified in the wireless infrastructure of a wireless hybrid network in order to achieve the best performances. Several strategies are proposed. Section 2 presents the routing protocol as well as the testbed that was used for simulations. Several strategies for mobility notification frame transmission are compared in section 3. Finally in section 4, we propose and compare several mechanisms to reduce the signaling overhead in the wireless hybrid network.

\section{Wireless Hybrid Network}

The global architecture is composed of a wireless infrastructure network extended by a general Mobile Ad-Hoc Network (MANet). This differs from the work done in [4] since the infrastructure network is wireless and the ad hoc connectivity may extend further than two hops. In this article, we focus on the ad hoc nodes mobility notification process within the wireless infrastructure network and do not compare routing strategies in hybrid networks. Such a study may be found in [10].

Several micro-mobility protocols such as Cellular IP, Hawaii, Hierarchical Mobile IP or Edge Mobility have been proposed for wire infrastructure networks. Their main tasks are to efficiently manage intra-domain routing, enabling mobiles to perform fast handoffs between Base Stations, as well as to provide a paging service. Several surveys and comparative studies have been published in [2,3]. In the rest of this article, we will only consider Cellular IP [9] as it is largely deployed and has been the subject of an ad hoc extension in many proposals $[8,1]$. The protocol is presented here in a version that has been slightly modified for use in a wireless infrastructure network and in in- 
teraction with a separate ad hoc routing protocol which is used to extend the infrastructure coverage.

Every $0.2 \mathrm{~s}$, mobile nodes broadcast ad hoc packets which contain the list of the mobile neighbors, other mobiles and Base Stations, and the identity of the Base Station the mobile has chosen to attach to. These packets play both the role of control packets for the ad hoc routing protocol and route update for the CIP-like protocol.

Infrastructure nodes also act like Base Stations as they communicate through a wireless medium and participate to ad hoc routing through the broadcast of ad hoc packets every $0.2 \mathrm{~s}$. These ad hoc packets contain a list of mobile neighbors and advertise them as a Base Station. Here again, these ad hoc packets play both the role of control packets to the ad hoc routing protocol and BS advertisement for the CIP-like protocol.

Mobility notification is implicitly initiated by a mobile. As a Base Station receives an ad hoc packet from an attached mobile, it transforms the ad hoc packet into a CIP-like route update packet and forwards it to the infrastructure network Gateway through its up-link neighbor. In an infrastructure node, reception of a mobile ad hoc packet updates the route entry for this mobile and reception of a route update packet updates the downgoing route to the mobile with the last forwarder as next hop. An infrastructure route has a lifetime of $0.5 \mathrm{~s}$. By default, if no specific route is known, a data packet is forwarded toward the infrastructure Gateway. Mobile nodes always transmit their data packet to their Base Station.

The up-link neighbor of an infrastructure node is the father's node in the CIP-like routing tree. This tree, routed at the gateway and spanning the infrastructure network, is created by diffusion of a gateway advertisement packet, periodically emitted by the gateway and forwarded by all infrastructure nodes.

We performed our simulations using the network simulator NS-2 ${ }^{1}$. The topology network used consists of 9 wireless infrastructure nodes with the addition of 2 to 64 mobile nodes which move according to the Random Waypoint Mobility model. The maximum speed of the mobile nodes has been set to $50 \mathrm{~m} / \mathrm{s}$. Constant Bit Rate data flows of 5 packets of 500 bytes per second are simulated between the mobile nodes.

\section{Comparing the Route Update strategies}

The radio medium is far from being similar to a classical wire medium such as the Ethernet one and differs in several aspects. Wireless links are pervasive and not isolated due to the broadcast nature of the medium. In consequence, the topology of the infrastructure network is not efficiently mapped into a routing tree as it is in Cellular IP. Another difference between wired and wireless 
links is the medium transmission quality and efficiency as bandwidth is smaller and latency is greater in air. These differences lead to the fact that some design aspects chosen during the development of Cellular IP may no longer be appropriate. For example, the choice to transmit route update packets in unicast increases reliability but also medium occupancy and prevents from routing along efficient paths.

\subsection{Acknowledged broadcast}

The IEEE 802.11 distributed coordination function (DCF) basically provides two MAC level modes for frame transmissions: unicast and broadcast modes. Unicast frames require acknowledgment from the receiver. The lack of acknowledgement reception causes the retransmission of the frame until the transmission succeeds or the maximum retransmission count is exceeded. Unicast frames can also be protected, especially against hidden node situations, by using a Request to send - Clear to send (RTS-CTS) exchange prior to frame transmission. Broadcasted frames are neither protected by RTS-CTS, nor acknowledged. Therefore, correct reception cannot be guaranteed. But, if the same data rate is used, broadcasted frames are far more efficient when transmitting information to a set of neighbor nodes. Both strategies present advantages for route update messages transmission. Unicast mode favors reliability and can help maintaining an accurate view of the network topology. Broadcasted frames favor speed and allow the spread of the topology updates faster. Mixing the two approaches to obtain a more reliable broadcast could be profitable. Nevertheless, acknowledging broadcasted frames is not straightforward as multiple acknowledgements from multiple receivers would collide. Therefore, acknowledging broadcasted frames requires the selection of one particular neighbor to acknowledge frames, as if the message was transmitted in unicast mode and every other mode were in a promiscuous reception mode. Wireless hybrid networks provide a hierarchical organization removing the need for dynamic election of the only neighbor acknwoledging frames that arises in a pure ad hoc context. When a node emits a route update message, it is destined to its father in the routing tree. If other infrastructure nodes can overhear this message, they will also benefit from this information, adding a route to the mobile and enabling a shortcut in the tree routing scheme of Cellular IP. In the following paragraphs, we will compare the results we obtained for the three possible strategies for route update transmission: using unicast transmission, broadcast or acknowledged broadcast. In order to correctly study the differences between this three transmission modes, we will keep the tree routing scheme of Cellular IP and avoid taking the advantages offered by the broadcast and acknowledged broadcast modes. 


\subsection{Simulation Results}

First of all, we will compare three strategies at the MAC-level for route update messages transmission. Unicast mode includes RTS-CTS excahnge and acknowledgements. Acknowledges broadcast suppresses RTS-CTS exchange and broadcast suppresses both mechanisms.

With Cellular IP, each node will regularly emit ad hoc packets, each Base Station will regularly forward route update packets and relay gateway advert isement packets. Signaling can represent a high load when the network gets dense. Signaling packets nevertheless carry useful information and should neither be lost, nor be delayed too much. Losing route updates will result in many routing table inconsistencies and delaying these packets too much will result in outdated information in the routing tables. We need to find the correct balance between network load and informations accuracy.

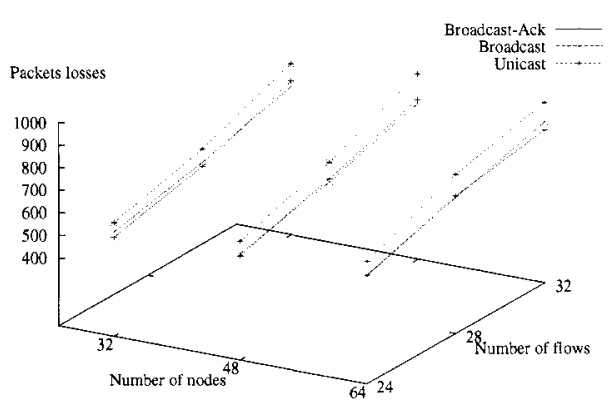

Figure 1. Losses of CBR packets: route disappearance

Figure 1 presents the losses of data packets due to the absence of route towards the destination in the whole network for configurations where the medium is overloaded (more than 16 CBR data flows). This situation arises when a route has been deleted due to timeout and the new route has not yet been discovered or propagated. Data packets are forwarded to the Gateway, which is the root of the infrastructure network, that drops the packet. Unicast transmission of route update messages leads to the highest data packet loss rate, due to the delay introduced by the protection of signaling frames. Routes are not refreshed in time and routing tables entries disappear.

On the opposite, Figure 2 presents the losses of data packets due to retransmissions by the infrastructure nodes. This situation happens whenever a mobile has moved but the routing entry in the infrastructure network still references the old base station. In this situation, broadcast transmission of route 
update frames leads to the highest loss number due to the low reliability of the signaling messages transmission.

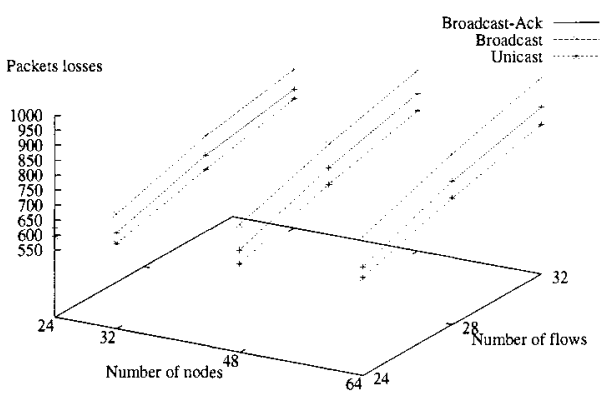

Figure 2. Losses of CBR packets: wrong routing entry

Both strategies present advantages as well as drawbacks and it is difficult to determine which method will yield optimal results. Acknowledged broadcast is an "in the middle" approach and could lead to better overall results in the end. Figure 3 represents the total number of packets that have been correctly transmitted in each of the simulations performed. As soon as the data flows saturate the medium, broadcast mode outperforms unicast mode by $25 \%$ in the best case. Unicast transmission of Route Update messages always results in the poorest performance, followed by acknowledged broadcast and broadcast.

The number of packets successfully delivered is highly dependent on the network load. When the network is not overloaded, the performances of the three strategies are equivalent. Then, when the network capacity is exceeded, the overhead introduced by the route update transmission mode results in a difference in the number of packets successfully transmitted. As transmitting a packet in broadcast mode requires less time than transmitting the same packet in unicast mode, the medium capacity is exceeded later with broadcast route update packets. Finally, performances become equivalent again when the network is overloaded regardless of the transmission mode.

\section{Optimization of the mobility notification}

From the results of sections 3, we can deduce that the main challenge to improve data traffic delivery is to reduce the radio medium utilization. We have to reconsider the experimental protocol described in section 2 in order to lower the number of control packets its use requires. 


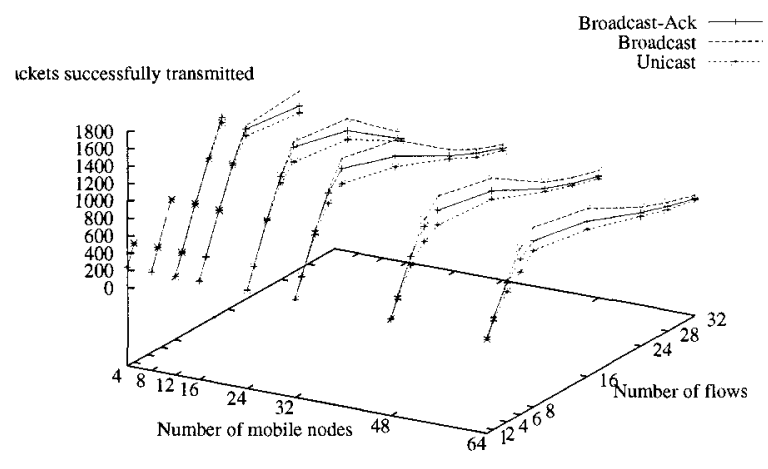

Figure 3. Number of CBR packets correctly received

\subsection{Differential Route updates}

Frequent route updates are necessary while the mobile performs a handoff. A new route has to be set up in the infrastructure network as fast as possible in order to avoid misrouting and losing of data packets. A first route update packet must be sent after the mobile's handoff to create the route. This sending must be repeated in short successive intervals of time in order to prevent the loss of the previous route update packets as their delivery is not reliable. After the route setup and while the mobile remains connected to the Base Station, frequent updates are no longer needed. The time interval between two consecutive updates may be increased in order to lower the number of control packets. However, the frequency of ad hoc packet emission may not be reduced as cellular stillness is far from meaning ad hoc stillness. In consequence, only a subset of the ad hoc packets may be forwarded by Base Stations as route update packets.

We introduce a flag in ad hoc packets to notify the Base Station whether or not the packet must be forwarded in the infrastructure as a route update. The flag is set by the mobile as it is the one which initiates the handoff. After a handoff, the first period between two consecutive route-update is $0.2 \mathrm{~s}$ and this period is increased by a factor 1.5 for each consecutive route-update. We call this mechanism differential route update.

\subsection{Nack route}

While using differential route update mechanism, route update emission intervals may be larger than the ad hoc packets period. This means that the 
link between the mobile and its Base Station is refreshed at a higher rate than the infrastructure down-going route to the mobile. In consequence, the Base Station may notice a mobile's handoff long before the mobile's route times out in the infrastructure. This route reminiscence may lead to incorrect routing and data packet loss. In order to prevent this phenomenon, a Base Station may, as soon as it has detected a mobile has left its cell, discard the route towards this mobile in the infrastructure. As routing incoherence between different infrastructure routers may lead to routing loops, it is not enough for the Base Station to only discard the route on its own. It should notify all infrastructure routers concerned by the now out-dated route, that it is no longer valid. This is realized by the Base Station emitting a route delete packet which is forwarded to the infrastructure Gateway along the same path as route update packets. This packet discards the mobile's route in the infrastructure routers on its path. We call this mechanism nack route.

\subsection{Nack only}

If we carry on trying to reduce control traffic to its extreme, we can completely avoid multiple route update emissions after a mobile handoff and send only one. This strategy is optimistic in the sense that it makes the supposition that route update packets may not be lost in the infrastructure. If it is lost, there will be no infrastructure route to the mobile and data packets will be dropped. Since only one route update is sent for each handoff and no refreshment is further performed, infrastructure routes have an infinite lifetime. To invalid an old route after a mobile handoff, Base Stations send a route delete packet, as explained in the previous section. This strategy sounds far from reliable as only one route update loss has catastrophic consequences, but it has the advantage of drastically reducing the control traffic.

\subsection{Simulation Results}

Simulations have been carried out for broadcast, unicast as well as acknowledged broadcast route update transmission modes. Usually, using the optimizations described above in these three transmission modes provides similar results when evaluating the optimizations performances. Even if the numbers of packets successfully transmitted are not the same, the phenomenons described below are the same for the three modes. Therefore we will only present figures for one single transmission mode. Simulations show that the number of nodes in the network has a much lower influence on overall performance when compared to the number of data flows in the network. Therefore, for readability, we will only present results for networks of 64 mobile nodes, as results are also similar when considering fewer nodes. 


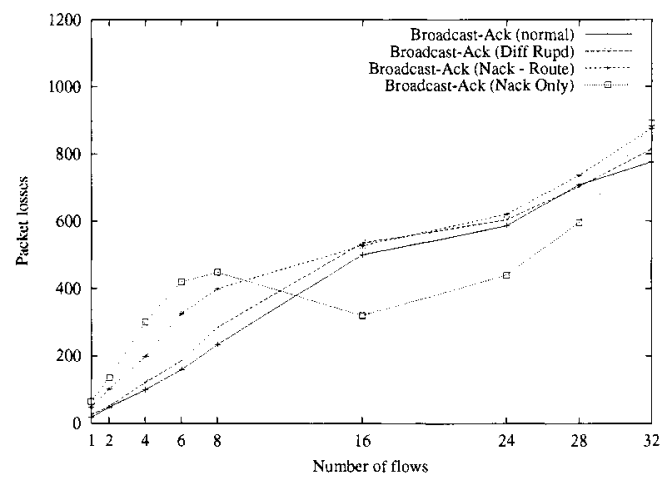

Figure 4. Amount of data packets lost due to route disappearance (64 mobiles; acknowledged broadcast)

To evaluate the performance of the different routing strategies, we will look at the influence of the different optimizations on the routing tables validity. Figure 4 represents the number of data packet losses due to route disappearance, i.e., no route exists to reach the destination mobile, neither in the Base Station to which the sender is attached to, nor in the Gateway. These drops occur when a route expires in the whole network before the new route to the mobile has been propagated. When the medium is lightly loaded, optimizations seem to increase the number of drops at the Gateway. But as soon as the medium gets overloaded, the Nack Only optimization which reduces the load due to signaling packets, is highly efficient. As route update messages represent a high load, the medium saturation point is postponed. However, losing a route update message has a much greater impact with Nack Only optimization, that's why performance collapse again under a high data load.

However, Figure 5 shows the number of data packets lost due to outdated entries in the routing tables. The Base Station in charge of the receiver tries to forward the data frame to the mobile, gets no acknowledgment in return, concludes there has been a collision and retries to forward the frame until the retransmission counter is exceeded. These losses are also due to repeated collisions resulting in retransmissions but analysis of the trace files show that this cause is marginal compared to mobility-related losses. These results show that the more optimizations we add, the more the routes are outdated. This is due to the increasing delay between two route update packets sending, resulting in a increasing route timeout.

Optimizations described here lead to the same kind of discussion as the one on the different ways to transmit route update messages. One one hand, we will try to send as few route update frames as possible, but 


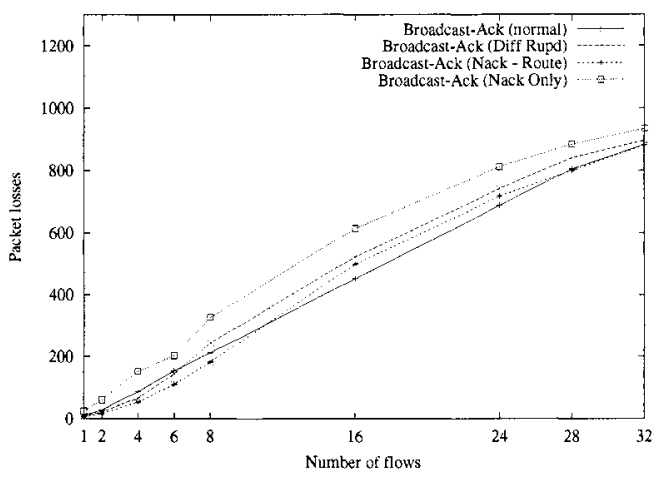

Figure 5. Amount of data packets lost due to errors in routing tables (64 mobiles; acknowledged broadcast)

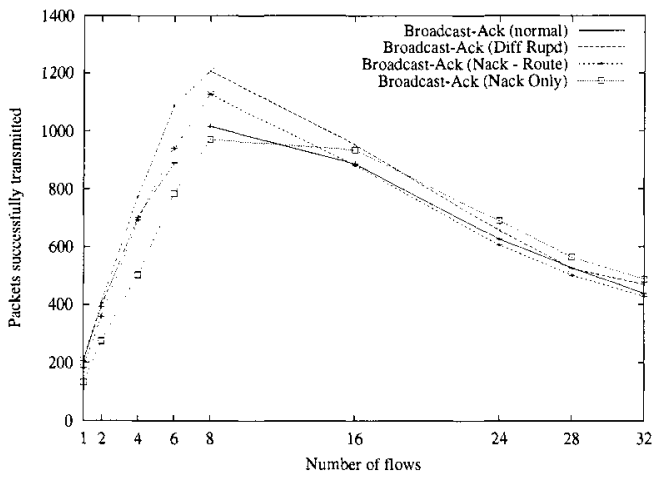

Figure 6. Amount of data packets successfully transmitted (64 mobiles; acknowledged broadcast) 
we will be less reactive to mobility and on the other hand, over-occupying the medium will delay data packets and raise the number of packets lost due to collisions. Figure 6 represents the total amount of data packets successfully delivered. If simple Differential Route Update mode always shows good performances, other mode performances are load-dependent. Nack Only optimization is rather good when the medium is overloaded but represents a loss of performance when there is no real need for saving bandwidth.

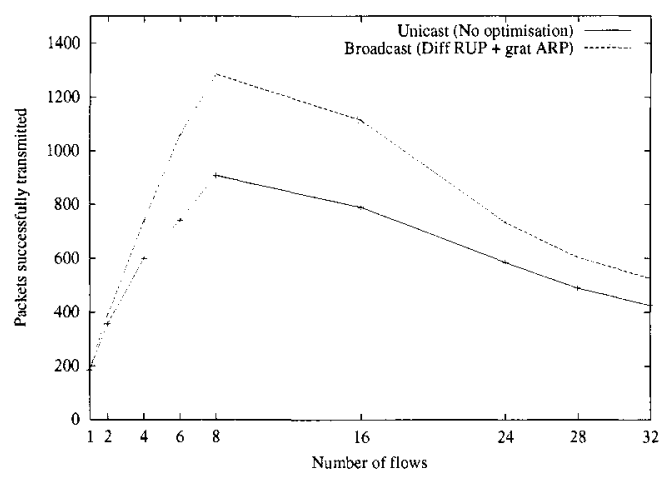

Figure 7. Number of packets correctly transmitted in normal mode and with optimisations

To conclude this study, Figure 7 shows the total number of data packets correctly transmitted by a regular Cellular IP compared to a modified version in which route update packets are broadcasted and the differential route update optimization is activated. This optimized Cellular IP leads to the best performances, enhancing the overall data throughput by up to $40 \%$.

\section{Conclusion}

In this article, we presented several possible modifications of the Cellular IP protocol for enhancing its performance in a wireless hybrid context. These modifications, concerning routing as well as MAC layer, show that network performances can be increased by up to $40 \%$. These results can still be enhanced, for example by implementing optimizations of the routing scheme related to the broadcast transmission of signaling frames. These results especially show how micro-mobility protocols derived from wired protocols are inadequate in a wireless context. Mechanisms that have proved themselves worthy in regular networks such as reliable unicast transmission should be left aside in most situations.

Performance should not be expected to get similar to those obtained in wired networks. Nevertheless, the efficiency of wireless hybrid networks can be in- 
creased. This work shows that the key issue regarding network performance is the network load. This load can be decreased on one hand by reducing the global signaling volume as studied here and on the other hand by designing suited radio interfaces and medium access protocols. The separated signaling channel mechanism, allocating a particular frequency, time slot or CDMA code to control traffic, widely used in cellular telephony networks might lead to further performance enhancements. Nevertheless, actual wireless hardwares do not allow this due to the long channel switching delay.

\section{References}

[1] R. Bhan, A. Croswell, K. Dedhia, W. Lin, M. Manteo, S. Merchant, A. Pajjuri, and J. Thomas. Adding ad hoc Network Capabilities to Cellular IP. Technical report, www.columbia.edu.

[2] A. Campbell, J. Gomez, S. Kim, Z. Turanyi, C-Y. Wan, and A. Valk. Comparison of ip micromobility protocols. IEEE Wireless Communications, 9(1):72-82, February 2002.

[3] A. Campbell and J. Gomez-Castellanos. Ip micromobility protocols. ACM SIGMOBILE Mobile Computing and Communications Review, 4(4):45-53, October 2000.

[4] R-S. Chang, W-Y. Chen, and Y-F. Wen. Hybrid wireless network protocols. IEEE Transactions on Vehicular Technology, 52(4):1099-1109, July 2003.

[5] H-C. Chao and C-Y. Huang. Micro-mobility mechanism for smooth handoffs in an integrated ad-hoc and cellular ipv6 network under high-speed movement. IEEE Transactions on Vehicular Technology, 52(6):1576-1593, November 2003.

[6] G. Chelius and É. Fleury. Design of a hybrid routing architecture. In IEEE MWCN, Singapore, November 2003. IEEE Communications Society.

[7] Y-Z. Huang. Dynamic adaptive routing for heterogeneous wireless network. Master's thesis, National Central University, West Lafayette, USA, 2001.

[8] V. Typpö. Mobility within Wireless Ad Hoc Networks: Towards Hybrid Wireless Multihop Networks. Master's thesis, VTT Electronics and University of Oulu, Finland, 2001.

[9] A. Valkó. Cellular ip - a new approach to internet host mobility. ACM Computer Communication Review, 29(1):50-65, January 1999.

[10] C. Wijting and R. Prasad. Evaluation of mobile ad-hoc network techniques in a cellular network. In IEEE VTC, pages 1025-1029, 2000. 